\title{
Are the spoken durations of rare words longer than those of common words?
}

\author{
GINA GEFFEN and MARY A. LUSZCZ \\ Centre for Neuroscience and Psychology Discipline \\ Flinders University of South Australia, Adelaide 5042, Australia
}

\begin{abstract}
The effects of word frequency, word length, and practice were examined in oral productions of subjects reading lists of 25 rare or common monosyllabic words. Articulation and pause durations, their ratio, and total reading durations were derived from recordings of subjects' speech. Recorded speech was sampled at $10 \mathrm{kHz}$, and a criterion of eight times the mean noise level was used to classify productions as articulation or pause. Lists of highfrequency words were read more quickly than lists of low-frequency words. No differences were observed in the articulation component. Pause duration was greater for rare than for common words. The ratio of pause to articulation varied with length and word type. No differences were found for high-frequency words, but the ratio of five-letter words was significantly greater than that of three or four-letter rare words. Results were discussed in relation to the nature and locus of the word-frequency effect. Criteria for defining and measuring speech productions were also raised.
\end{abstract}

Common words are more easily accessed from memory and recognized in print than are rare words. This has been found in tasks that involve naming objects (Oldfield \& Wingfield, 1965), reading isolated words (Berry, 1971 ; Forster \& Chambers, 1973), making lexical decisions (Forster \& Chambers, 1973), and reading word lists (Geffen, Stierman, \& Tildesley, 1979). In the last study, the durations of both pause and articulation were separately measured while subjects read aloud lists of 20 words. Geffen et al. (1979) concluded that the frequency level of a word has a major effect during an early stage when words are first analyzed, since pause time rather than articulation time was affected by word frequency.

In contrast, Wright (1979) claimed to show that spoken durations of rare words are longer than those of common words. The results of Wright compared to those of Geffen et al. (1979) are inconclusive, if not inconsistent. Methodological differences between the studies preclude a reconciliation. For instance, Geffen et al. (1979) measured pause and articulation durations of lists of low-, middle-, and high-frequency monosyllabic and polysyllabic word lists, and Wright (1979) measured only reading times of lists of monosyllabic words. This measure included pauses between words (Wright, Note 1). We report a replication of Wright's first experiment in which both pause and articulation durations were measured and analyzed. In accordance with the Geffen et al. (1979) results, it was expected that frequency effects would be found in the pause rather than the articulation

This work represents a collaborative effort; first authorship will be shared on a rotating basis. The work was supported by a grant from the A.R.G.C. We are grateful to Kustas Tiivas for developing the software, to Ken McFarland for testing criterion levels, and to Jo Wale for running subjects. component of reading. Further, we extended Wright's experiment to examine the effect of practice on reading word lists that differ in length and frequency of occurrence. It was hypothesized that, over trials, rereading of lists would reduce differences between high-and lowfrequency words.

\section{METHOD}

\section{Materials}

Six sets of single-syllable rare (frequencies $\leqslant 3$ per million) and common (frequencies $\geqslant 100$ per million) words of three, four, or five letters were selected from the Kucera and Francis (1967) word count. Words were selected (as in Wright, 1979) if (1) they appeared in Webster's New World Dictionary, (2) they could not be decomposed into a stem and a simple ending, (3) their pronunciation was not ambiguous (e.g., lead), and (4) they were not obscene. Lists of 78, 170, and 97 common and 91,265 , and 223 rare three-, four-, and five-letter words, respectively, were obtained.

The sets of words were sampled randomly without replacement for each subject to generate six 25 -word lists, one each of three-, four-, and five-letter rare and common words. Each list was typed double-spaced, lowercase, in a single column centered on white paper, for easy reading by subjects.

Subjects

Seven psychology students (two female) at Flinders University of South Australia acted as subjects. Each was tested individually and paid a nominal sum for participating.

\section{Design}

A frequency (2) by length (3) by trials (3) factorial design with repeated measures on all factors was employed. Order of occurrence of rare and common lists of different lengths was balanced across subjects. Each list was read five times in succession, with the first trial serving as familiarization and the final, as a buffer.

\section{Procedure and Apparatus}

Subjects were seated at a table in front of a microphone used 
to record their speech. The position for reading material was marked on the table, and papers on which the lists had been typed were placed in a predetermined order. During a practice trial, recording levels were adjusted for each individual. Subjects were instructed to read each list five times, as quickly and accurately as possible, each time beginning as soon as possible after they heard the "start" tone $(3 \mathrm{kHz})$. This tone was presented immediately at the start of a list to indicate that reading should commence. The experimenter pressed a button on a tone box that automatically recorded the tones on one channel of a Revox two-channel tape recorder. Subjects' speech was recorded on the other channel.

Tape recordings of subjects' pause and articulation times were analyzed by a PDP- $11 / 34$ computer program. An analogto-digital converter sampled the filtered $(5 \mathrm{kHz})$ speech recordings at $10 \mathrm{kHz}$ and produced a file stored on disk for further analyses. The experimenter-generated signals on one channel of the tape recorder regulated the sampling of the speech on the other channel. A mean noise level $(\mathrm{MNL}=$ sum of the absolute differences of adjacent points, at .1-msec intervals, divided by the total number of points in the sample) was calculated separately for each file. Discrimination of articulation vs. pause times was effected by adopting a criterion of eight times the MNL. This criterion was empirically determined by varying the volume of the speech input of three randomly selected speech samples from three subjects and examining the effect of selecting different criteria for speech time. The minimum-and most stable criterion appeared to be eight times the MNL. Below this, the pause time component was added to speech time. At 16 times the MNL, speech time was counted as pauses. At eight times the MNL, similar speech times were obtained regardless of input volume. Between four and eight times the MNL, a great deal of variability was found. Thus, points of less than eight times the MNL were designated "pause"; points greater than or equal to the criterion and "pauses" of less than $50 \mathrm{msec}$ duration were regarded as "articulation." Therefore, intraword pauses of greater than $50 \mathrm{msec}$ (which should be rare) were classified as pause duration.

\section{RESULTS}

From the files described above, four dependent mea- sures were calculated for analysis for each list: pause time, articulation time, the sum of these components or reading time (Figure 1 depicts these results), and the ratio of pause to articulation time. Each of these measures was subjected to a 2 (frequency) by 3 (length) by 3 (trials) analysis of variance. Only data from Trials 2 , 3 , and 4 were analyzed.

The reading time measure indicated that highfrequency lists were read more quickly than lowfrequency lists (mean $=10.0$ vs. $13.0 \mathrm{sec}$, respectively) $[F(1,6)=10.95, \mathrm{p}<.05]$, in agreement with previous results (Geffen et al., 1979; Wright, 1979). Similar to Wright's results, the slopes of the lines relating reading duration to letter length differed for high- and lowfrequency words. The reading time measure, which includes pauses, may be too gross, in that apparent interactions (e.g., Wright's result shown in Figure $1 \mathrm{~b}$ and our result, Figure 1d) were not significant due to low power and/or great variability. We therefore analyzed the articulation and pause time components separately. Articulation time produced no significant effects. Pause times and the ratio of pause to articulation times proved to be more informative measures. The frequency effect noted in the combined measure seemed to be attributable to differences in pause duration for common $($ mean $=5.9)$ relative to rare $($ mean $=7.5 \mathrm{sec})$ words $[F(1,6)=8.29, p<.05]$. The ratio measure showed a main effect of length $[F(2,12)=5.28, p<.05]$ and an interaction of Frequency by Length $[F(2,12)=4.02$, $\mathrm{p}<.05]$. Newman-Keuls post hoc tests showed no differences for high-frequency words. However, rare fiveletter words yielded a significantly higher ratio than three- or four-letter rare words, which did not differ from each other. The nature of the two-way interaction is shown in Figure 2. a.

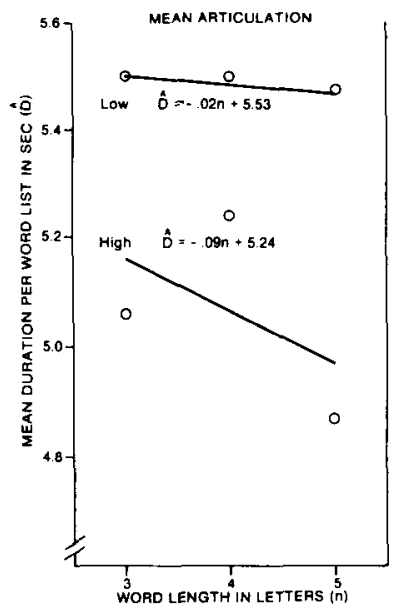

b.

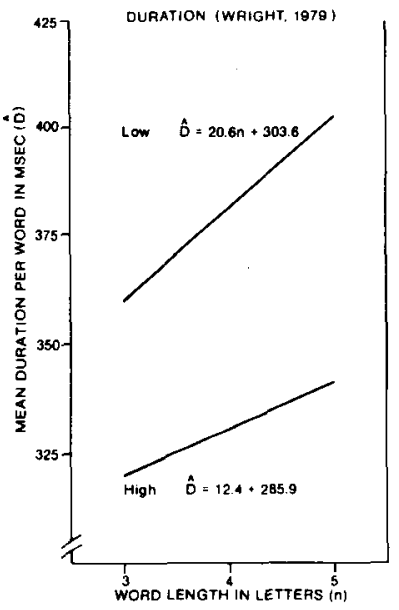

c.

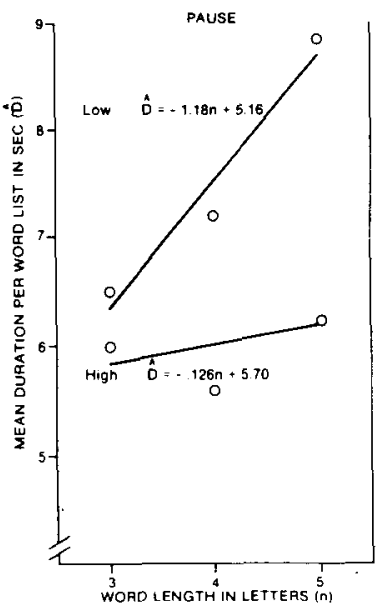

d.

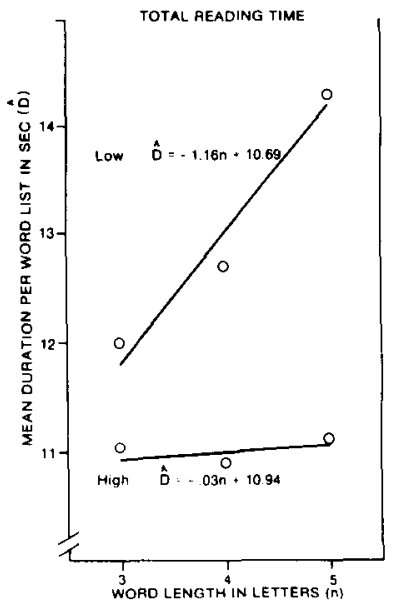

Figure 1. (a) Mean articulation duration (in seconds) of lists, with seven subjects and 25-word lists read three times for each point, as a function of word length. (b) The mean duration results of Wright (1979) are reproduced. Wright obtained duration per word in milliseconds by dividing total reading time by 25 , since his lists also contained 25 words. (c) Mean pause times (in seconds) in a 25 -word list (three lists and seven subjects), as a function of word length. (d) Reading time (in seconds) per 25-word lists (three lists and seven subjects), as a function of word length. 


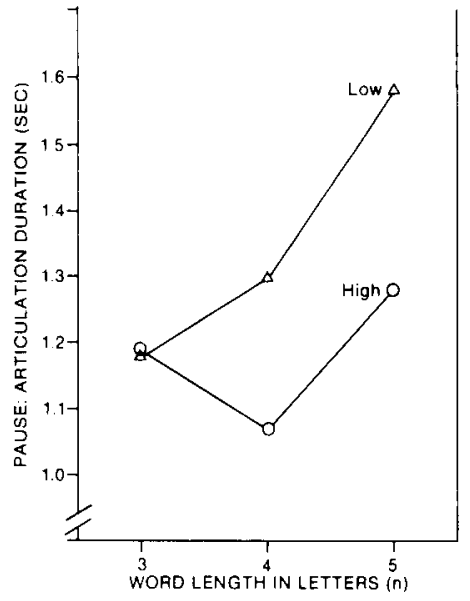

Figure 2. Ratio of pause to articulation time as a function of word length for high-and low-frequency words. Each point is the mean for seven subjects reading 25 -word lists three times.

\section{DISCUSSION}

The frequency of usage of words affected the pause times rather than duration of articulation or total reading duration. The results of Geffen et al. (1979) have been replicated, as have those of Wright (1979) for total reading time (compare Figures $1 \mathrm{~b}$ and $1 \mathrm{~d}$ ). However, it is clear from the separate analysis of articulation and pause components of total reading time that effects attributed to total reading time were in fact due to variations in pause patterns characteristic of high- vs. lowfrequency words (compare Figures 1c and 1d). When low-frequency words take marginally longer to articulate than high-frequency words (see Figure 1a), the basis for increases in total reading time is clearly related to increments in pauses.

Both phonetic and semantic features distinguish the words and have been cited as explanations for observed differences. According to Wright (1979), rare words are more difficult to say (therefore, they take longer to articulate), but according to Geffen et al. (1979), rare words are harder to locate in the internal lexicon (therefore, we pause in uttering them). Our data cast doubt on an articulatory basis for word-frequency effects. Our interpretation of the functional significance of pauses still requires substantiation, but it is clearly this component of oral reading that varies with word frequency.

It is crucial to define the relationship between the measure one is taking and the underlying variable that the measure is supposed to represent. This is particularly important if the measure can be altered by shifts in criterion (e.g., of speech vs. pause) that accord with theoretical preferences. The criterion of eight times the MNL appears to be a suitable measure that is sensitive to both word length and word frequency. Further indirect evidence of the validity of criteria adopted for distinguishing between speech and pause time was the finding of Geffen et al. (1979) that delayed speech feedback produced much longer articulation durations but had a smaller effect on pauses.

\section{REFERENCE NOTE}

1. Wright, C. E. Personal communication, August 24, 1981.

\section{REFERENCES}

BERRY, C. Advanced frequency information and verbal response time. Psychonomic Science, 1971, 23, 151-152.

Forster, K. I., \& Chambers, S. Lexical access and naming time. Journal of Verbal Learning and Verbal Behavior, 1973, 12, 627-635.

Gerten, G., Stierman, I., \& Tildegley, P. The effect of word length and frequency on articulation and pausing during delayed auditory feedback. Language and Speech, 1979, 22, 191-199.

Kučera, H., \& Francis, W. N. Computational analysis of present-day American English. Providence, R.I: Brown University Press, 1967.

OldField, R. C., \& Wingfield, A. Response latencies in naming objects. Quarterly Journal of Experimental Psychology, 1965, 17, 273-281.

Wright, C. E. Duration differences between rare and common words and their implications for the interpretation of word frequency effects. Memory \& Cognition, 1979, 7, 411-419.

(Received for publication August 16, 1982; revision accepted August 25, 1982.) 